\title{
THE SPACE OF FUNCTIONS OF BOUNDED VARIATION AND CERTAIN GENERAL SPACES*
}

\author{
BY \\ C. RAYMOND ADAMS
}

1. Introduction. With usual interpretations of addition, multiplication by a real number, and equality, the space $(B V)$ of functions $x(t)$ of bounded variation on the interval $\dagger(0,1)$ is a vector space. $\neq$ Various writers $\S$ have introduced a metric by defining the norm $\|x\|$ and the distance function $(x, y)$ as follows: $\|x\|=|x(0)|+T_{0}{ }^{1}(x)$, where $T_{0}^{1}(x)$ designates the total variation of $x(t)$ on $(0,1) ;(x, y)=\|x-y\|$. Thus metrized, $(B V)$ is a Banach space (or space of type $(B))$; so also are its subspaces $(C B V)$, composed of the continuous functions in $(B V)$, and $(A C)$, composed of the absolutely continuous functions. Although with this metric $(A C)$ is a separable space, neither $(B V)$ nor $(C B V)$ is.

Indeed\| Banach and Mazur, loc. cit., have noted that ("ersichtlich") every set in $(B V)$ whose cardinal number is less than that of the continuum is non-dense in $(B V)$. The reasoning may run as follows: let $x_{h}(t) \quad(0<h<1)$ be the characteristic function of the point $t=h$; then for $h_{1} \neq h_{2}$ we have $\left(x_{h_{1}}, x_{h_{2}}\right)=T_{6}^{1}\left(x_{h_{1}}-x_{h_{2}}\right)=4$, and there exists a continuum of disjoint spheres $\mathbb{T}$ $K\left(x_{h}, 1\right) \subset K(\theta, 3)$; hence an arbitrary sphere $K(x, r) \subset(B V)$ contains a continuum of disjoint spheres $K\left(r x_{h} / 3+x, r / 3\right)$. For $(C B V)$, as well as for its subspace $(C S)$ of continuous singular functions, the same property can be demonstrated in a similar way by the aid of some results due to Wiener and

* Presented to the Society, April 11, 1936, under a slightly different title; received by the editors March 10, 1936.

$\dagger$ The interval $(0,1)$ is always understood to mean $0 \leqq t \leqq 1$.

$\ddagger$ In this paper we generally employ the terminology and notation of Banach, Théorie des Opérations Linéaires, Warsaw, 1932.

$\S$ These include Hahn, Über Folgen linearer Operationen, Monatshefte für Mathematik und Physik, vol. 32 (1922), pp. 3-88; Plessner, Eine Kennzeichnung der totalstetiger Funktionen, Crelle's Journal, vol. 160 (1929), pp. 26-32; Hildebrandt, Linear functional transformations in general spaces, Bulletin of the American Mathematical Society, vol. 37 (1931), pp. 185-212; Banach and Mazur, Zur Theorie der linearen Dimension, Studia Mathematica, vol. 4 (1933), pp. 100-112; and Gergen, Note on linear operations in functions of bounded variation (abstract), Bulletin of the American Mathematical Society, vol. 40 (1934), p. 657.

$\| \mathrm{We}$ are indebted to J. A. Clarkson for a suggestion in connection with the contents of this paragraph, which was added August 24, 1936.

II In general the zero-element of a space will be designated by $\theta$. 
Young.* Perhaps the simplest procedure is to define $x(t)$ on $\left(0, \frac{1}{2}\right)$ as Wiener and Young's modification (loc. cit., §6) of Cantor's ternary function, on $\left(\frac{1}{2}, 1\right)$ as $x(1-t)$, and elsewhere as the (continuous) periodic continuation. Then, allowing $x_{h}(t)$ to stand for $x(t+h)$, we have $\left(x_{h_{1}}, x_{h_{2}}\right)=\left(x_{h_{2}-h_{1}}, x\right)=2 T_{0}^{1}(x)=2$ for $0<h_{1}<h_{2}<1$.

One would expect the lack of separability to lead to difficulties in handling certain questions concerning $(B V)$ and $(C B V)$, including that of determining the form of the general linear functional $\dagger$ (i.e., real-valued linear operation) on $(B V)$.

In recent papers by Clarkson, Lewy, and the present writer $\ddagger$ consideration has been given to a sequence of functions $x_{n}(t) \quad(n=1,2, \cdots)$, of bounded variation, subject to the following conditions: (i) $x_{n}(t)$ tends everywhere on $(0,1)$ to a limit function $x(t) \varepsilon(B V)$, as $n \rightarrow \infty$; and (ii) $T_{0}^{1}\left(x_{n}\right) \rightarrow T_{0}{ }^{1}(x)$ [or (ii') $L_{0}^{1}\left(x_{n}\right) \rightarrow L_{0}^{1}(x)$, where $L_{0}^{1}(x)$ denotes the length, in the sense of Peano, of $x(t)$ on $(0,1)]$. To describe this situation the notation $x_{n}(t)-v \rightarrow x(t)$ $\left[x_{n}(t)-l \rightarrow x(t)\right]$ was introduced, the symbol $-v \rightarrow[-l \rightarrow]$ being read "converges in variation" ["converges in length"]. These studies naturally lead one to contemplate the possibility of defining a metric for $(B V)$ in such a manner that convergence in the metric will have a meaning as close as possible to convergence in variation or to convergence in length. From this standpoint the following four metrics all invite attention:

$$
\begin{aligned}
& (x, y)_{1}=\int_{0}^{1}|x(t)-y(t)| d t+\left|T_{0}^{1}(x)-T_{0}^{1}(y)\right|, \\
& (x, y)_{2}=\int_{0}^{1}|x(t)-y(t)| d t+\left|L_{0}^{1}(x)-L_{0}^{1}(y)\right|, \\
& (x, y)_{3}=\sup _{0 \leqq t \leqq 1}|x(t)-y(t)|+\left|T_{0}^{1}(x)-T_{0}^{1}(y)\right|, \\
& (x, y)_{4}=\sup _{0 \leqq t \leqq 1}|x(t)-y(t)|+\left|L_{0}{ }^{1}(x)-L_{0}{ }^{1}(y)\right| .
\end{aligned}
$$

Among these metrics the relations $(x, y)_{1} \leqq(x, y)_{3},(x, y)_{2} \leqq(x, y)_{4}$ for every $x, y_{\varepsilon}(B V)$ are apparent. With the metric $(x, y)_{3}\left[(x, y)_{4}\right]$ each point of the set $(D B V)$ (i.e., each discontinuous function of bounded variation) is the center

* Wiener and Young, The total variation of $g(x+h)-g(x)$, these Transactions, vol. 35 (1933), pp. 327-340. An independent proof of the non-separability of $(C B V)$ and $(C S)$ has been constructed by A.P. Morse on the basis of results and methods contained in his paper On convergence in variation, to appear shortly in these Transactions.

$\dagger$ Cf. Gergen, loc. cit.; his form for the functional is $f(x)=\int_{0}^{1} K(x, t) d x(t)$.

$\ddagger$ Adams and Clarkson, On convergence in variation, Bulletin of the American Mathematical Society, vol. 40 (1934), pp. 413-417; Adams and Lewy, On convergence in length, Duke Mathematical Journal, vol. 1 (1935), pp. 19-26. Hereinafter these papers will be referred to as I and II respectively. 
of a sphere containing no points* of $(C B V)$, in marked contrast to the fact that a sequence of continuous functions may converge in variation [length] to a discontinuous function.

We observe that the relation $x_{n}-v \rightarrow x$ implies $\left(x_{n}, x\right)_{1} \rightarrow 0$; for the functions $x_{n}(t)$ are uniformly bounded and tend to $x(t)$ everywhere on $(0,1)$. Conversely, when $x(t)$ is continuous on $(0,1)$, the relation $\dagger\left(x_{n}, x\right)_{1} \rightarrow 0$ implies $x_{n}-v \rightarrow x$; this may readily be proved by aid of Theorem 3 of $I$ and the fact that every subsequence of $x_{n}(t)$ contains a subsequence which converges almost everywhere. If, however, we have $\left(x_{n}, x\right)_{1} \rightarrow 0$ and $x(t)$ is discontinuous at even a single point, $x_{n}(t)$ may fail to converge to $x(t)$ at every point in $(0,1)$; this may be shown by a trivial modification of a familiar type of example illustrating a sequence which converges in the mean without converging anywhere.f Thus, although in the space $(C B V)$ the two kinds of convergence are equivalent, in the space $(B V)$ convergence in the metric $(x, y)_{1}$ is a somewhat weaker condition than convergence in variation.

Precisely the same statements hold for the implications, or lack of the same, between convergence in length and convergence in the metric $(x, y)_{2}$.

As for the relationship between convergence in the metrics $(x, y)_{1}$ and $(x, y)_{2}$, we see that when $x(t)$ is continuous on $(0,1),\left(x_{n}, x\right)_{2} \rightarrow 0$ implies $x_{n}-l \rightarrow x$, which by Theorem 1 of II implies $x_{n}-v \rightarrow x$, which implies $\left(x_{n}, x\right)_{1} \rightarrow 0$. The condition $\left(x_{n}, x\right)_{2} \rightarrow 0$ fails to imply $\left(x_{n}, x\right)_{1} \rightarrow 0$, however, when $x(t)$ has even a single discontinuity; this may be seen by slightly altering an example given in $\$ 5$ of I, merely introducing a suitable jump in the limit function (there called $f_{0}$ ) at $t=1$. From Theorem 1 of II it is clear that, even in the space $(A C),\left(x_{n}, x\right)_{1} \rightarrow 0$ does not imply $\left(x_{n}, x\right)_{2} \rightarrow 0$.

With either of the metrics $(x, y)_{3}$ and $(x, y)_{4}$ the spaces $(B V),(C B V)$, and $(A C)$ are not complete and not even locally compact. To verify this one need consider only such a simple example as a sequence of polygonal functions all having the same total variation and the same length on $(0,1)$ and converging uniformly to $x(t) \equiv 0$.

With the metric $(x, y)_{1}$, however, every bounded portion of $(B V)$ is compact. For the set of functions $x(t)$ satisfying the condition $(x, \theta)_{1}=\int_{0}^{1}|x(t)| d t$

* See the corollary to Theorem 4 of I and the corollary to Theorem 2 of II.

$\dagger$ Each continuous function of bounded variation is an element of the metric space $(B V)$, metrized with $(x, y)_{1}$; on the other hand an element of $(D B V)$ is not a single discontinuous function but a class of such, any two functions in this class being equal almost everywhere and having the same total variation. Here and throughout the remainder of the Introduction, if the sequence $x, x_{1}$, $x_{2}, \cdots$ contains discontinuous functions, it is really non-essential whether one regards the relation $\left(x_{n}, x\right)_{1} \rightarrow 0\left[\left(x_{n}, x\right)_{2} \rightarrow 0\right]$ as a condition on a sequence of single functions or on a sequence of classes of (metrically equal) functions.

‡ See, for instance, Titchmarsh, The Theory of Functions, Oxford, 1932, p. 390, Example (ii). 
$+T_{0}{ }^{1}(x) \leqq M$ is clearly of uniformly bounded variation and hence is readily seen to be uniformly bounded. By a well known theorem of Helly, ${ }^{*}$ this set contains a sequence $x_{n}(t)$ converging everywhere to a function $x(t) \varepsilon(B V)$, and by Lebesgue's convergence theorem $x_{n}(t)$ converges in the mean to $x(t)$. But, the sequence $T_{0}{ }^{1}\left(x_{n}\right)$ being bounded, it contains a subsequence $T_{0}^{1}\left(x_{n_{i}}\right)$ convergent to a limit $\tau$; and by aid of the semi-continuity property, $\dagger$ lim inf $T_{0}{ }^{1}\left(x_{n_{i}}\right) \geqq T_{0}{ }^{1}(x)$, we infer $\tau \geqq T_{0}{ }^{1}(x)$. Redefining $x(0)$, if necessary, so that $T_{0}^{1}(x)=\tau$, we have $\left(x_{n_{i}}, x\right)_{1} \rightarrow 0$ and the compactness is proved. The completeness of the space follows at once. Since uniform boundedness of the lengths of a set of functions implies that condition on their total variations, $\dagger$ and since length also enjoys the semi-continuity property, $\dagger$ the same sort of reasoning can be employed to establish the same results for $(B V)$ metrized with $(x, y)_{2}$.

With either metric $(x, y)_{1}$ or $(x, y)_{2}$ the space $(B V)$ is separable; indeed there exists a denumerable subset of $(A C)$ which is dense in $(B V)$. For each metric this assertion may be established as follows. If $x(t)$ is any function in $(B V)$, it can be approximated $\dagger$ in the metric by an inscribed polygonal function $y(t)$. But any such function can obviously be approximated in the metric by a continuous polygonal function $z(t)$ whose "corners" have rational coordinates. Clearly the functions $z(t)$ are a denumerable subset of $(A C)$.

These considerations make it apparent that, of the four metrics in question, the first two are to be preferred above the last two. Of $(x, y)_{1}$ and $(x, y)_{2}$, only the former enjoys the homogeneity property $(a x, a y)=|a| \cdot(x, y)$, for every real number $a$ and every $x, y \varepsilon(B V)$, a property whose importance will become amply evident in $\$ 2$. Henceforth in this paper the metric $(x, y)_{1}$ will always be employed for $(B V)$ unless the contrary is expressly stated. From an example given in $\S 5$ of $I$ it is clear that convergence in this metric, even when we restrict ourselves to the space $(A C)$, is not in general an additive property. We therefore devote $\$ 2$ to the development of certain elementary properties of spaces which are more weakly conditioned by postulate than are the spaces considered in Banach's book (loc. cit.). Although motivated by our present needs, the discussion contained in $\$ 2$ may be of some intrinsic interest, dealing as it does with spaces in which the translation property $(x, y)=(x-y, \theta)$ is absent, the relation $\lim \left(x_{n}+y_{n}\right)=\lim x_{n}+\lim y_{n}$ does not in general hold, continuity of an additive and homogeneous operation at one point does not imply continuity everywhere else, and continuity of such an operation over a space does not imply uniform continuity. In $\$ 3$ we make a few remarks of a

* Helly, Über lineare Funktionaloperationen, Sitzungsberichte der Wiener Akademie, vol. 121, IIa (1912), p. 283.

† See $\$ 2$ of II. 
more or less geometric nature concerning the space $(B V)$. In $\$ 4$ we consider functionals on certain general spaces, and in $\$ 5$ continuous functionals on the space $(B V)$. So far as we are aware, this is the first instance in which the general form of an additive and uniformly continuous functional is determined for a space not of Banach type.

The interesting question as to the general form of an additive functional which is continuous without being uniformly continuous now arises, apparently for the first time. This problem seems to call for a method of treatment essentially different from that introduced by F. Riesz $z^{*}$ in 1909, and at present we have nothing to contribute to its solution.

2. Certain general spaces. Consideration of $(B V)$ metrized with the distance function $(x, y)_{1}$ immediately focusses attention upon the meaning of the term equality in vector and metric spaces. The desirability of treating this notion postulationally has been remarked, both in print $\dagger$ and otherwise, by various persons. We do not propose to concern ourselves here with postulate theory and in particular shall not investigate questions of independence, but it is essential that our assumptions be clearly understood. For that reason we begin with definitions of vector and metric spaces, placing special emphasis on the notion of equality in the two cases and making a sharp distinction between vector equality and metric equality.

It may be worthwhile at the outset to state our own viewpoint, which is essentially as follows: for a given space the choice of a distance function imposes a definition of metric equality; with this meaning for equality the space may not be a vector space; nevertheless, with a suitable definition of vector equality, it may become a vector space; there is no logical reason why these two kinds of equality must necessarily be the same.

Oneł might wish to metrize, for example, a space of periodic functions having the same period, in such a way that two functions differing only in phase constant would be indistinguishable (i.e., metrically equal); whereas, when two functions were to be added, a distinction (provided by vector inequality) would have to be made between any two functions not identical. Our viewpoint may sometimes be useful in physics; in fact the "new non-

* F. Riesz, Sur les opérations fonctionnelles linéaires, Comptes Rendus, Paris, vol. 149 (1909), pp. 974-977.

† See Michal, A critique of the postulate systems for abstract vector spaces and abstract Hilbert spaces (abstract), Bulletin of the American Mathematical Society, vol. 39 (1933), p. 881; Taylor, $A$ reduced set of postulates for abstract Hilbert space, ibid., vol. 41 (1935), pp. 439-448, especially footnote on p. 439; and Highberg and Taylor, An independent set of postulates for abstract linear spaces (abstract), ibid., vol. 41 (1935), p. 802.

$\ddagger$ The addition (August 24,1936 ) of this paragraph was prompted by Koopman's review, cited below. 
relativistic point of view" of Dirac* in quantum mechanics is, to say the least, susceptible of an interpretation bearing a close analogy to ours. $\mathrm{He}$ now uses the term "state" to refer to the condition of a dynamical system at one instant of time, and considers the aggregate of states of a particular system as constituting a vector space. In this space we understand the element to be a single state, equality between states meaning identity and being analogous to our vector equality; and the law of combination is a "superposition" of two states the result of which is a third state whose relationship to the first two is expressible linearly, with complex numbers for coefficients. At the same time his "further assumption that by superposing a state with itself" one can form "only the original state over again" seems to imply an indistinguishability (between $a \psi$ and $\psi$ ) analogous to our metric equality.

Definition 1. A class $E$ of elements is said to constitute a vector space when the following postulates are satisfied: (1) to each ordered pair of elements $x, y_{\varepsilon} E$ there corresponds an element of $E$ designated by $x+y$ and called the sum of $x$ and $y$; (2) to each real number $a$ and each element $x_{\varepsilon} E$ there corresponds an element of $E$ designated by $a x$ and called the product of $x$ by $a$; (3) there exists a relation called vector equality and designated by the symbol $=$ such that if $x, y$ is any ordered pair of elements in $E$, either $x$ bears this relation to $y$ (in which case we write $x=y$ ) or it does not (in which case we write $x \neq y$ ); (4) the relation $=$ is reflexive (i.e., $x=x$ for every $x \varepsilon E$ ), symmetric (i.e., $x=y$ implies $y=x$ ), and transitive (i.e., $x=y$ and $y=z$ together imply $x=z$ ); and (5) with respect to this relation $=$, the sum and product fulfill the conditions $x+y=y+x, x+(y+z)=(x+y)+z, x+y=x+z$ implies $y=z, a(x+y)=a x+a y,(a+b) x=a x+b x, a(b x)=(a b) x, 1 x=x$, where $a, b$ are any real numbers and $x, y, z$ any elements in $E$.

We recall that in a vector space $E$ there exists a zero-element $\theta$ (unique in the sense determined by vector equality) having the properties: $x+\theta=x$ for every $x_{\varepsilon} E ; a \theta=\theta$ for every real number $a$.

Definition 2. A class $E$ of elements is said to constitute a metric space when the following postulates are satisfied: (1) to each ordered pair of elements $x, y_{\varepsilon} E$ there corresponds a real number called the distance from $x$ to $y$ and designated by $(x, y) ;(2)$ there exists a relation called metric equality (or metric equivalence) and designated by the symbol $\cong$ such that if $x, y$ is any ordered pair of elements in $E$, either $x$ stands in this relation to $y$ (we then

* Dirac, The Principles of Quantum Mechanics, 2d edition, Oxford, 1935, especially Chapters I, II. In the language (punctuation included) of his reviewer (Koopman, Bulletin of the Americar Mathematical Society, vol. 42 (1936), p. 472), "The point of view is to regard the aggregate of states of a system somehow as forming a linear vector space, the elements of which both are and are not the states (there is an indeterminate phase factor!), and ...." 
write $x \cong y$ ) or it does not (we then write $x$ not $\cong y$ ); (3) the relation $\cong$ is reflexive, symmetric, and transitive; and (4) with respect to this relation $\cong$, the distance function satisfies the conditions $(x, y)=0$ for $x \cong y,(x, y)>0$ for $x$ not $\cong y,(x, y)=(y, x),(x, z) \leqq(x, y)+(y, z)$ (the triangle postulate), for any elements $x, y, z \varepsilon E$.

We observe that in a metric space $E$, for any $x, y, z \varepsilon E$,

$$
x \cong y \quad \text { implies } \quad(x, z)=(y, z) .
$$

This follows at once since we have

$$
(x, z) \leqq(x, y)+(y, z)=(y, z) \leqq(y, x)+(x, z)=(x, z) .
$$

The statement that $U(x)$ is an operation defined on a space $E$ with counter-domain in a space $E^{\prime}$ (or more briefly, on $E$ to $E^{\prime}$ ) is commonly understood, and we shall so understand it, to mean that to each element $x_{\varepsilon} E$ there corresponds an element $U(x)_{\varepsilon} E^{\prime}$. The following postulate, which appears to have been tacitly assumed by some writers, we impose explicitly.

Postulate 1. An operation $U(x)$ shall be said to be defined on a vector space $E$ to a like space $E^{\prime}$ only if $x=y_{\varepsilon} E$ implies $U(x)=U(y)_{\varepsilon} E^{\prime}$.

For our present purposes it seems desirable (although it may not always be so) to impose also

Postulate 2. In a vector metric space $E$ the relation $x=y$ shall imply $x \cong y$, for $x, y_{\varepsilon} E$.

Definition 3. In a vector metric space we define the norm of any element $x$, written $\|x\|$, as $(x, \theta)$.

Definition 4. An operation $U(x)$ on a metric space $E$ to a like space shall be called continuous at $x$ when for $x_{n}, x_{\varepsilon} E$

$$
\left(x_{n}, x\right) \rightarrow 0 \quad \text { implies } \quad\left(U\left(x_{n}\right), U(x)\right) \rightarrow 0 ;
$$

continuous on $E$ when it is continuous at every point of $E$; and uniformly continuous on $E$ when for every $x_{n}, y_{n} \varepsilon E$

$$
\left(x_{n}, y_{n}\right) \rightarrow 0 \quad \text { implies } \quad\left(U\left(x_{n}\right), U\left(y_{n}\right)\right) \rightarrow 0 .
$$

THEOREM 2.1.* Let $U(x)$ be an operation on a metric space $E$ to a like space. If $U(x)$ is continuous at a point $x \varepsilon E$ and $x \cong y$, a necessary and sufficient condition that $U(x)$ be continuous at $y$ is $U(x) \cong U(y)$.

* If operations not everywhere continuous were to be considered, one would probably find it desirable to replace this theorem by the following analogue of Postulate 1: an operation $U(x)$ shall be said to be defined on a metric space $E$ to a like space $E^{\prime}$ only if $x \cong y_{\varepsilon} E$ implies $U(x) \cong U(y)_{\varepsilon} E^{\prime}$. 
Proof. For the sufficiency, let $x_{n}$ be any sequence such that $\left(x_{n}, y\right) \rightarrow 0$; then $\left(x_{n}, x\right) \rightarrow 0$, which implies $\left(U\left(x_{n}\right), U(x)\right)=\left(U\left(x_{n}\right), U(y)\right) \rightarrow 0$. For the necessity, we have both $\left(x_{n}, y\right) \rightarrow 0$ and $\left(x_{n}, x\right) \rightarrow 0$; hence both $\left(U\left(x_{n}\right), U(y)\right) \rightarrow 0$ and $\left(U\left(x_{n}\right), U(x)\right) \rightarrow 0$, and $(U(x), U(y))=0$ follows from the triangle postulate.

The space of real numbers, with the usual interpretations, is a Banach space; we designate it by (A).

Definition 5. A vector metric space $E$ in which the metric has the additional property $\dagger$

$$
(a x, a y)=|a| \cdot(x, y)
$$

for every $a \varepsilon(A)$; every $x, y \varepsilon E$,

shall be called a space of type $\left(\alpha^{*}\right)$.

It may be observed that in a space $E$ of type $\left(\alpha^{*}\right)$, the relation $a x \cong y$ $\left(0 \neq a \varepsilon(A) ; x, y_{\varepsilon} E\right)$ implies $x \cong(1 / a) y$. Addition of metric equalities is not in general permissible, however, even when the space is more strongly conditioned (as it will be later when spaces of types $\left(\alpha^{* *}\right)$ and $(\alpha)$ are introduced).

Definition 6. An operation $U(x)$ on a vector metric space $E$ to a like space shall be called strongly additive when

$$
U(x+y)=U(x)+U(y)
$$

for every $x, y \varepsilon E$;

weakly additive when

$$
U(x+y) \cong U(x)+U(y)
$$

for every $x, y \varepsilon E$;

strongly homogeneous when

$$
U(a x)=a U(x)
$$

for every $a_{\varepsilon}(A)$, every $x \varepsilon E$;

and weakly homogeneous when

$$
U(a x) \cong a U(x)
$$

for every $a_{\varepsilon}(A)$, every $x \varepsilon E$.

THEOREM 2.2. Let $U(x)$ be an operation on a space $E$ of type $\left(\alpha^{*}\right)$ to a like space. $\ddagger$ If $U(x)$ is weakly homogeneous and uniformly continuous on $E$, it satisfies a Lipschitz condition

$$
(U(x), U(y)) \leqq M \cdot(x, y) . \quad \text { for every } x, y \varepsilon E .
$$

Conversely, it is clear that if $U(x)$ satisfies (2.3), it is uniformly continuous on $E$.

Proof. In the absence of such a constant $M$, there would exist sequences

$\dagger$ The independence of this property is shown by $(B V)$ metrized with $(x, y)_{2}$.

$\ddagger$ It may be noted that the following less restrictive hypotheses on the spaces are sufficient for the validity of this theorem: each space to be one in which multiplication of an element by a real number is possible (i.e., merely property (2), Definition 1 , of a vector space), and to be a metric space in which the metric has the homogeneity property (2.2). 
$x_{n}, y_{n} \varepsilon E$ such that $\left(U\left(x_{n}\right), U\left(y_{n}\right)\right)>n \cdot\left(x_{n}, y_{n}\right)$, with $\left(x_{n}, y_{n}\right)>0$ by Theorem 2.1. Designating by $X_{n}, Y_{n}$ respectively the elements

$$
\frac{1}{n\left(x_{n}, y_{n}\right)} x_{n}, \quad \frac{1}{n\left(x_{n}, y_{n}\right)} y_{n} \varepsilon E,
$$

we have by (2.2)

$$
\left(X_{n}, Y_{n}\right)=\frac{1}{n} \rightarrow 0 .
$$

Since $U(x)$ is uniformly continuous, this relation must imply $\left(U\left(X_{n}\right), U\left(Y_{n}\right)\right)$ $\rightarrow 0$. But, $U(x)$ being weakly homogeneous, we have by (2.1) for all $n$,

$$
\begin{aligned}
\left(U\left(X_{n}\right), U\left(Y_{n}\right)\right) & =\left(\frac{1}{n\left(x_{n}, y_{n}\right)} U\left(x_{n}\right), \frac{1}{n\left(x_{n}, y_{n}\right)} U\left(y_{n}\right)\right) \\
& =\frac{1}{n\left(x_{n}, y_{n}\right)}\left(U\left(x_{n}\right), U\left(y_{n}\right)\right)>1 .
\end{aligned}
$$

From this contradiction follows condition (2.3).

ThEOREM 2.3. Let $U(x)$ be an operation on a space $E$ of type $\left(\alpha^{*}\right)$ to a like space. If $U(x)$ is strongly additive and weakly homogeneous on $E$ and continuous at $\theta$, it satisfies a Lipschitz condition at the point $\theta$,

$$
\|U(x)\| \leqq M_{\theta} \cdot\|x\| \quad \text { for every } x \varepsilon E .
$$

Conversely, it is clear that if $U(x)$ satisfies (2.4), it is continuous at $\theta$.

Proof. From the strong additivity of $U(x)$ we have $U(x)=U(x+\theta)$ $=U(x)+U(\theta)$; hence $U(\theta)=\theta^{\prime}$, the zero-element of the counter-domain. We may then employ the same kind of argument as in the proof of Theorem 2.2 to obtain the condition (2.4).

CoRollary. Let $U(x)$ be an operation on a space $E$ of type $\left(\alpha^{*}\right)$ to a space of type $(F)$ (sense of Banach). If $U(x)$ is additive and homogeneous on $E$ and continuous at $\theta$, it satisfies the condition

$$
(U(x), U(y)) \leqq M_{\theta} \cdot\|x-y\| \quad \text { for every } x, y \varepsilon E .
$$

Definition 7. Let $U(x)$ be an operation on a space $E$ of type $\left(\alpha^{*}\right)$ to a like space. If $U(x)$ satisfies a Lipschitz condition (2.3), we designate by $\bmod _{E} U$, the modulus of the operation $U(x)$ on $E$, the smallest number $M$ for which (2.3) holds. If $U(x)$ satisfies the condition (2.4), we designate by $\|U\|_{E}$, the norm of the operation $U(x)$ on $E$, the smallest number $M_{\theta}$ for which (2.4) is fulfilled. 
If $U(x)$ is weakly homogeneous, it is clear from (2.1) and (2.2) that for every $a_{\varepsilon}(A)$ and every $x, y_{\varepsilon} E$ we have

$$
\frac{(U(a x), U(a y))}{(a x, a y)}=\frac{(a U(x), a U(y))}{|a| \cdot(x, y)}=\frac{(U(x), U(y))}{(x, y)},
$$

whence

$$
\begin{aligned}
\bmod _{E} U & =\sup _{x, y_{E} E,(x, y)=1}(U(x), U(y)), \\
\|U\|_{E} & =\sup _{x \in E,\|x\|=1}\|U(x)\| .
\end{aligned}
$$

We clearly have $\|U\|_{E} \leqq \bmod _{E} U$, whether both norm and modulus exist finite or otherwise.

Definition 8. A space $E$ of type $\left(\alpha^{*}\right)$ in which the metric has the additional property

$$
\left(a_{n} x, x\right) \rightarrow 0
$$

for every real sequence $a_{n} \rightarrow 1$ and every $x \varepsilon E$, shall be called a space of type $\left(\alpha^{* *}\right)$.

The following properties of this type of space may be noted: for $a_{n}, a_{\varepsilon}(A)$ we have

$$
\begin{array}{rlr}
a_{n} \rightarrow 0 \text { implies }\left\|a_{n} x\right\| & =\left|a_{n}\right| \cdot\|x\| \rightarrow 0 & \text { for every } x \varepsilon E ; \\
\left(x_{n}, x\right) \rightarrow 0 \text { implies }\left(a x_{n}, a x\right) & =|a| \cdot\left(x_{n}, x\right) \rightarrow 0 & \text { for every } a \\
a_{n} \rightarrow a \neq 0 \text { implies }\left(a_{n} x, a x\right) & =|a| \cdot\left(\frac{a_{n}}{a} x, x\right) \rightarrow 0 & \text { for every } x \varepsilon E ;
\end{array}
$$

whence, by aid of the triangle postulate, it may readily be proved that for $a_{m}, a, b_{n}, b_{\varepsilon}(A)$ and $x_{p}, x, y_{q}, y_{\varepsilon} E$, the relations $a_{m} \rightarrow a, b_{n} \rightarrow b,\left(x_{p}, x\right) \rightarrow 0$, $\left(y_{q}, y\right) \rightarrow 0$ imply

$$
\left(a_{m} x_{p}, b_{n} y_{q}\right) \rightarrow(a x, b y) \quad \text { as } m, n, p, q \rightarrow \infty .
$$

TheOREm 2.4. Let $U(x)$ be an operation on a space $E$ of type $\left(\alpha^{* *}\right)$ to a like space. If $U(x)$ is strongly additive [weakly additive and $U(-x) \cong-U(x)$ for every $\left.x_{\varepsilon} E\right]$ and continuous on $E$, it is weakly homogeneous.

Proof. From the hypotheses it follows easily that $U(r x)=r U(x)$ $[U(r x) \cong r U(x)$ ] for every rational $r$ [every rational $r \neq 0$ ]. Let $a$ be any irrational number and $r_{n}$ a sequence of rationals converging to $a$. By (2.10) we have, for every $x_{\varepsilon} E,\left(r_{n} x, a x\right) \rightarrow 0$; in view of the continuity this implies $\left(U\left(r_{n} x\right), U(a x)\right) \rightarrow 0$, or $\left(r_{n} U(x), U(a x)\right) \rightarrow 0$. But since the counter-domain has 
property (2.7), $r_{n} \rightarrow a$ also implies $\left(r_{n} U(x), a U(x)\right) \rightarrow 0$. From the triangle postulate we conclude $U(a x) \cong a U(x)$. In a similar manner continuity enables us to infer from the bracketed hypotheses $U(\theta) \cong \theta^{\prime}$, which completes the proof.

Definition 9. A space $E$ of type $\left(\alpha^{*}\right)$ in which the metric has the additional property $(x, y) \leqq\|x-y\|$ for every $x, y_{\varepsilon} E$ shall be called a space of type $(\alpha)$.

We observe that every vector metric space has the property $|\|x\|-\|y\||$ $\leqq(x, y)$; hence a space of type $(\alpha)$ satisfies the condition $|\|x\|-\|y\|| \leqq\|x-y\|$, which implies

$$
\|x+y\| \leqq\|x\|+\|y\| .
$$

A space of type $(\alpha)$ is also of type $\left(\alpha^{* *}\right)$.

Remarks. (I) Every "normed vector space" (sense of Banach) is of type $(\alpha)$. (II) The space $(B V)$ becomes a vector space when we interpret addition and multiplication by a constant in the usual way and $x=y$ as meaning that the functions $x(t)$ and $y(t)$ are identical on the interval $0 \leqq t \leqq 1$. Upon introducing the distance function $(x, y)_{1}$ and interpreting $x \cong y$ as meaning that the functions $x(t)$ and $y(t)$ are equal almost everywhere and have the same total variation on $0 \leqq t \leqq 1$, it becomes a vector metric space, in fact a space of type $(\alpha)$. Henceforth these interpretations are always to be understood when we speak of "the space $(B V)$." It is clear that $(B V)$ is not a "normed vector space." (III) Every Banach space (or space of type $(B)$ ) is a complete space of type $(\alpha)$. (IV) The class of Banach spaces is the meet of the class of Fréchet spaces (or spaces of type $(F)$ ) and the class of complete spaces of type $\left(\alpha^{*}\right)$ in which $=$ and $\cong$ have the same meaning. (V) A space [complete space] of type $\left(\alpha^{*}\right)$ is a "normed vector space" [Banach space] if and only if the metric has the additional property $(x, y)=\|x-y\|$ and there is no distinction between metric and vector equality.

3. Some properties of the space $(B V)$. Let $x, y$ be any two points in $(B V)$ with $x \neq y$; then the set of points

$$
z=h x+(1-h) y
$$$$
(0 \leqq h \leqq 1)
$$

constitutes, by definition, the segment $x y$. If $h_{0}, h_{1}, \cdots, h_{n}$ is any finite set of values of $h$ satisfying the condition $0=h_{0}<h_{1}<\cdots<h_{n}=1$ and the corresponding $z$ 's are denoted by corresponding subscripts, we define the length of the segment $x y$ as the least upper bound of $\sum_{i=1}^{n}\left(z_{i-1}, z_{i}\right)$ for all such sets. With this terminology one may easily verify the following statements. A segment is a continuous curve; whence it follows that $(B V)$ is a connected space. 
Each segment $x y$ has a finite positive length which does not exceed $\int_{0}^{1}|x-y| d t+T_{0}^{1}(x-y)$. This bound for the length of a segment is attainable. A segment may have a continuum of multiple points (multiple in the sense determined by metric equality). When $x, y$ are on the surface of a sphere, the segment $x y$ may contain points outside the sphere.

4. Linear functionals on a space of type $(\alpha)$. By the term functional we mean an operation $U(x)$ on a space $E$ to the space $(A)$ of real numbers. Noting that for a functional the qualification of additivity and homogeneity by "strong" and "weak" loses its significance, we establish two theorems as follows.

TheOREM 4.1. Given a space $G$ of type $(\alpha)$ embedded in a space $E$ of the same type, and a functional $f(x)$ additive and homogeneous on $G$ and continuous on $G$ at $\theta$; then there exists a functional $F(x)$, additive and homogeneous on $E$ and continuous on $E$ at $\theta$, which satisfies the conditions $F(x)=f(x)$ for every $x_{\varepsilon} G$ and $\|F\|_{E}=\|f\|_{G}$.

Proof. For $x_{\varepsilon} E$ we set $p(x)=\|f\|_{G} \cdot\|x\|$ in the Hahn-Banach theorem on extension of functionals (Theorem 1, Banach, loc. cit., pp. 27-28). Then by (2.11) we have for $x, y_{\varepsilon} E, p(x+y) \leqq p(x)+p(y)$; and by (2.2) for $x \varepsilon E$ and $t \geqq 0, p(t x)=t p(x)$. $E$ and $G$ being vector spaces, $f(x)$ additive and homogeneous on $G$, and $f(x) \leqq p(x)$ on $G$, we conclude from the Hahn-Banach theorem the existence on $E$ of an additive and homogeneous functional $F(x)$ coinciding with $f(x)$ on $G$ and having the property $F(x) \leqq p(x)$ for every $x \varepsilon E$. It remains only to show that $F(x)$ is continuous on $E$ at $\theta$ and $\|F\|_{E}=\|f\|_{G}$. For any $x_{\varepsilon} E$ for which $F(x)$ is $\geqq 0$ we have $F(x) \leqq p(x)=\|f\|_{G} \cdot\|x\|$; for any $x_{\varepsilon} E$ for which $F(x)$ is $<0$ the homogeneity of $F(x)$ gives us $-F(x)=F(-x) \leqq p(-x)$ $=\|f\|_{G} \cdot\|x\|$. Hence $\|F\|_{E} \leqq\|f\|_{G}$, and the inequality is ruled out by the fact that $F(x)=f(x)$ on $G$.

THEOREM 4.2. For every $x_{0} \neq \theta$ in a space $E$ of type $(\alpha)$ there exists an additive and homogeneous functional $F(x)$, defined on $E$ and continuous at $\theta$, which is uniformly continuous on a subspace $G$ of type $(\alpha)$ containing $x_{0}$ and which satisfies the conditions $F\left(x_{0}\right)=\left\|x_{0}\right\|$ and $\|F\|_{E}=1$.

Proof. It suffices to designate by $G$ in Theorem 4.1 the set of elements $a x_{0}$, $a \varepsilon(A)$, and to set $f\left(a x_{0}\right)=a \cdot\left\|x_{0}\right\|$.

It is natural to raise the question as to whether Theorem 4.1 is the best that can be obtained; in particular, whether the following might be true: given an additive and uniformly continuous functional $f(x)$ defined on a space $G$ of type $(\alpha)$ embedded in a space $E$ of the same type, there exists an additive and uniformly continuous functional $F(x)$ defined on $E$, with 
$F(x)=f(x)$ for $x_{\varepsilon} G$ (and with or without an increase in the modulus). If this were so, it would imply that for every $x \neq \theta$ in a space $E$ of type $(\alpha)$ there exists an additive and uniformly continuous functional $F(x)$ defined on $E$, with $F\left(x_{0}\right)=\left\|x_{0}\right\|$. But $(B V)$ is a space of type $(\alpha)$, and by Theorem 5.2 every additive and uniformly continuous functional on $(B V)$ vanishes at $x_{0}$, where $x_{0}(t)=1$ for $t=0$ and is zero otherwise, although $\left\|x_{0}\right\|=1$. Hence the suggested extension is not always possible.

5. Functionals on the space $(B V)$. We first establish

Theorem 5.1. If $\alpha(t)$ is any summable function, the integral

$$
\int_{0}^{1} x(t) \alpha(t) d t
$$

defines on the space $(B V)$ a functional $f(x)$ which is additive, continuous, and homogeneous. The functionals generated by $\alpha_{1}(t)$ and $\alpha_{2}(t)$ are identical if and only if $\alpha_{1}(t)$ and $\alpha_{2}(t)$ are essentially the same* on $(0,1)$. The functional $f(x)$ is uniformly continuous on $(B V)$ if and only if $\alpha(t)$ is essentially bounded on $(0,1)$; in that case we have $\bmod _{(B V)} f=\operatorname{ess} \sup _{0 \leqq t \leqq 1}|\alpha(t)|$.

Proof. The existence, additivity, and homogeneity of $f(x)$ are apparent. To prove the continuity, let $x$ be any point $\varepsilon(B V)$ and $x_{n}$ any sequence such that $\left(x_{n}, x\right) \rightarrow 0$. There exists a number $K^{\prime}$ for which we have for all $n$

$$
\left(x_{n}, x\right)=\int_{0}^{1}\left|x_{n}-x\right| d t+\left|T_{0}^{1}\left(x_{n}\right)-T_{0}^{1}(x)\right| \leqq K^{\prime}
$$

hence for each $n$ there is at least one point $\bar{t}_{n}$ such that

$$
\left|x_{n}\left(\bar{t}_{n}\right)-x\left(\bar{t}_{n}\right)\right| \leqq K^{\prime} \text {. }
$$

But for each $n$ and all $t$ we have

$$
\left|x_{n}(t)-x_{n}\left(\bar{t}_{n}\right)\right| \leqq T_{0}^{1}\left(x_{n}\right),\left|x(t)-x\left(\bar{t}_{n}\right)\right| \leqq T_{0}^{1}(x), T_{0}{ }^{1}\left(x_{n}\right) \leqq T_{0}^{1}(x)+K^{\prime},
$$

whence for all $n$ and $t$

$$
\left|x_{n}(t)-x(t)\right| \leqq 2 T_{0}^{1}(x)+2 K^{\prime}=K .
$$

Moreover, given $\epsilon>0$ there exists $\delta>0$ such that $\int_{E} K|\alpha| d t<\epsilon$ whenever $m E<\delta$. Choosing $N$ so that $m E_{t}[|\alpha(t)|>N]<\delta$, we have

$$
\begin{aligned}
\left|f\left(x_{n}\right)-f(x)\right|=\left|f\left(x_{n}-x\right)\right| & \leqq\left[\int_{E}+\int_{C E}\right]\left|x_{n}-x\right| \cdot|\alpha| d t \\
& \leqq \epsilon+N \int_{0}^{1}\left|x_{n}-x\right| d t
\end{aligned}
$$

which is $<2 \epsilon$ for $n$ sufficiently large.

* That is, $\alpha_{1}(t)=\alpha_{2}(t)$ almost everywhere. 
It is clear that if $\alpha_{1}(t)$ and $\alpha_{2}(t)$ are essentially the same, they generate the same functional. On the other hand if the same functional is generated by $\alpha_{1}(t)$ and $\alpha_{2}(t)$, we may choose $x(t)=1$ for $0 \leqq t \leqq y,=0$ for $y<t \leqq 1(0 \leqq y \leqq 1)$ and obtain $\int_{0}^{y} \alpha_{1}(t) d t=\int_{0}^{y} \alpha_{2}(t) d t$ for all $y$. The essential equality of $\alpha_{1}(t)$ and $\alpha_{2}(t)$ then follows at once. This fact, in conjunction with the proof* of the following Theorem 5.2, shows that a uniformly continuous functional $f(x)$ cannot be generated by an $\alpha(t)$ which is not essentially bounded.

That $f(x)$ is uniformly continuous when $\alpha(t)$ is essentially bounded follows at once from (2.5); we have

$$
\bmod _{(B V)} f=\sup _{x, y \bullet(B V),(x, y)=1}|f(x)-f(y)| \leqq \operatorname{ess}_{0 \leqq t \leqq 1}|\alpha(t)|=B .
$$

To show that in this case $\bmod _{(B V)} f=B$, we may proceed as follows. Let $\epsilon(>0)$ be arbitrarily small and set

$$
m E_{1}[\alpha(t)>B-\epsilon]=k_{1}, \quad m E_{2}[\alpha(t)<\epsilon-B]=k_{2} .
$$

Then either $k_{1}$ or $k_{2}$ is $>0$; the cases being symmetrical, we consider that in which $k_{1}>0$. Choose $\delta$ to satisfy the condition $0<\delta<k_{1} \epsilon$ and inclose $E_{1}$ in an open set $O_{1}$ such that $k_{1} \leqq m O_{1} \leqq k_{1}+\delta$. From $O_{1}$ select a finite number of open intervals constituting a set $O_{2}$ such that $m O_{2} \geqq m O_{1}-\delta \geqq k_{1}-\delta$, and define $\phi(t)=1 / k_{1}$ for $t_{\varepsilon} O_{2},=0$ elsewhere. Then $\phi(t)$ is in $(B V)$ and we have

$$
\begin{gathered}
\int_{0}^{1} \phi(t) \alpha(t) d t \geqq\left(k_{1}-\delta\right) \frac{1}{k_{1}}(B-\epsilon)-\delta \frac{1}{k_{1}} B \geqq B-\epsilon-2 B \epsilon, \\
\int_{0}^{1} \phi(t) d t \leqq \frac{1}{k_{1}}\left(k_{1}+\delta\right) \leqq 1+\epsilon .
\end{gathered}
$$

Finally we set $x(t)=-y(t)=\phi(t) / 2$ for all $t$ in $(0,1)$; then $T_{0}{ }^{1}(x)=T_{0}{ }^{1}(y)$ and we have

$$
\begin{aligned}
|f(x)-f(y)| & =\left|\int_{0}^{1}(x-y) \alpha d t\right|=\int_{0}^{1} \phi(t) \alpha(t) d t \geqq B-\epsilon-2 B \epsilon, \\
(x, y) & =\int_{0}^{1}|x-y| d t=\int_{0}^{1} \phi(t) d t \leqq 1+\epsilon,
\end{aligned}
$$

whence

$$
|f(x)-f(y)| /(x, y) \geqq(B-\epsilon-2 B \epsilon) /(1+\epsilon) .
$$

Since the latter quantity is arbitrarily close to $B$, we conclude that $\bmod _{(B V)}$ $f=B$.

* See the italicized statement there. 
THEOREM 5.2. Every additive and uniformly continuous functional defined on the space $(B V)$ can be expressed in the form (5.1), with $\alpha(t)$ summable, bounded, and independent of $x(t)$; moreover we have $\|f\|_{(B V)} \leqq \int_{0}^{1}|\alpha(t)| d t \leqq$ $\bmod _{(B V)} f=B$, where $B=$ ess $\sup _{0 \leqq t \leqq 1}|\alpha(t)|$.

Proof. Let $f(x)$ be any additive and uniformly continuous functional defined on $(B V)$. We set

$$
\xi_{t}(u)=\left\{\begin{array}{l}
1 \text { for } 0 \leqq u \leqq t \\
0 \text { for } t<u \leqq 1
\end{array}\right.
$$

and

$$
f\left(\xi_{t}\right)=g(t)
$$

For $0 \leqq t_{1}<t_{2} \leqq 1$ we have

$$
\begin{aligned}
\mid g\left(t_{1}\right)- & g\left(t_{2}\right) \mid=\left(f\left(\xi_{t_{1}}\right), f\left(\xi_{t_{2}}\right)\right) \\
& \leqq M \cdot\left(\xi_{t_{1}}, \xi_{t_{2}}\right)=M \int_{0}^{1}\left(\xi_{t_{2}}-\xi_{t_{1}}\right) d t+M\left|T_{0}^{1}\left(\xi_{t_{2}}\right)-T_{0}^{1}\left(\xi_{t_{1}}\right)\right|
\end{aligned}
$$

where $M=\bmod _{(B V)} f$. For $t_{2}<1$ the second term $\cdot$ on the right vanishes, and we have $\left|g\left(t_{1}\right)-g\left(t_{2}\right)\right| \leqq M\left(t_{2}-t_{1}\right)$; i.e., $g(t)$ satisfies a Lipschitz condition on the open interval $0 \leqq t<1$ and is absolutely continuous there. We now define

$$
\bar{g}(t)= \begin{cases}g(t) & \text { for } 0 \leqq t<1 \\ g(1-0) & \text { for } t=1\end{cases}
$$

the existence of the integral $\int_{0}^{1} x(t) d \bar{g}$ is then assured for every $x_{\varepsilon}(B V)$.

Let $x(t)$ be any function in $(B V)$ vanishing at $t=0$. In view of a remark in $\$ 2$ of II, there exists a sequence of polygonal functions $p_{n}(t)(n=1,2,3, \cdots)$ inscribed in $x(t)$ and converging in variation to $x(t)$; for each $n$ the "corners" of $p_{n}(t)$ are determined by a set $S_{n}$ of points

$$
0=t_{0, n}<t_{1, n}<t_{2, n}<\cdots<t_{r(n), n}=1
$$

with $S_{n} \subset S_{n+1}$ for every $n$ and $S=\sum_{n=1}^{\infty} S_{n}$ everywhere dense in $(0,1)$. We define a step-function $z_{n}(t)$ associated with $p_{n}(t)$ as follows:

$$
\begin{aligned}
& z_{n}(t)=x\left(t_{i, n}\right) \text { for } t_{i-1, n}<t \leqq t_{i, n}, \text { all } i \text { and } n ; \\
& z_{n}(0)=0 .
\end{aligned}
$$

Then it is clear that $T_{0}^{1}\left(z_{n}\right)=T_{0}^{1}\left(p_{n}\right)$, whence $T_{0}{ }^{1}\left(z_{n}\right) \rightarrow T_{0}{ }^{1}(x)$ as $n \rightarrow \infty$. Moreover, since $x(t)$ is Riemann integrable on $(0,1), z_{n}(t)$ converges in the mean to $x(t)$. Hence we have $\left(z_{n}, x\right) \rightarrow 0$, which implies $f\left(z_{n}\right) \rightarrow f(x)$.

But $z_{n}(u)$ may be expressed in the form 


$$
z_{n}(u)=\sum_{i} x\left(t_{i, n}\right)\left[\xi_{t_{i, n}}(u)-\xi_{t_{i-1, n}}(u)\right]
$$

therefore, since $f(x)$ is additive, we must have

$$
\begin{aligned}
f\left(z_{n}\right) & =\sum_{i} x\left(t_{i, n}\right)\left[g\left(i_{i, n}\right)-g\left(t_{i-1, n}\right)\right] \\
& =\sum_{i} x\left(t_{i, n}\right)\left[\bar{g}\left(t_{i, n}\right)-\bar{g}\left(t_{i-1, n}\right)\right]+x(1)[g(1)-\bar{g}(1)] .
\end{aligned}
$$

Under the conditions in force we infer

$$
f(x)=\lim _{n \rightarrow \infty} f\left(z_{n}\right)=\int_{0}^{1} x(t) d \bar{g}+x(1)[g(1)-\bar{g}(1)],
$$

which is the form of the functional for every $x(t)$ vanishing at $t=0$.

Any function $x(t)$ not vanishing at $t=0$ can be expressed as the sum $x_{1}(t)+x_{2}(t)$ where

$$
x_{1}(t)=\left\{\begin{array}{ll}
x(t) & \text { for } 0<t \leqq 1, \\
0 \quad \text { for } t=0,
\end{array} \quad x_{2}(t)= \begin{cases}x(0) & \text { for } t=0, \\
0 & \text { for } 0<t \leqq 1,\end{cases}\right.
$$

and, $f(x)$ being additive, we must have $f(x)=f\left(x_{1}\right)+f\left(x_{2}\right)$. But $f(x)$ being continuous, it must assume the same value at any two points $x_{2}, x_{3}$ such that $x_{2} \cong x_{3}$. Choosing $x_{3}$ as follows:

$$
x_{3}(t)=\left\{\begin{array}{l}
{[x(0)] / 2 \text { for } t=1 / 2,} \\
0 \text { otherwise }
\end{array}\right.
$$

we clearly have $x_{2} \cong x_{3}$, while $f\left(x_{3}\right)$ is given by (5.3) and has the value zero. Hence $f(x)$ is given by (5.3) even when $x(0) \neq 0$.

Next consider the functions

$$
x_{4}(t)=\left\{\begin{array}{l}
0 \text { for } 0 \leqq t<1, \\
1 \text { for } t=1,
\end{array} \quad x_{5}(t)= \begin{cases}0 \quad \text { for } 0 \leqq t<1 \\
-1 \text { for } t=1\end{cases}\right.
$$

Since $x_{4} \cong x_{5}$, we must have $f\left(x_{4}\right)=f\left(x_{5}\right)$; hence by (5.3) we have $g(1)-\bar{g}(1)=$ $-[g(1)-\bar{g}(1)]$, whence $g(1)=\bar{g}(1)$ and (5.3) reduces to

$$
f(x)=\int_{0}^{1} x(t) d g \quad \text { for all } x \varepsilon(B V),
$$

where $g(t)$ is absolutely continuous on $0 \leqq t \leqq 1$. The form (5.1) for $f(x)$ is an immediate consequence, with $\alpha(t)$ bounded, since in fact $g(t)$ satisfies a Lipschitz condition on $0 \leqq t \leqq 1$. The evaluation of $\bmod _{(B V)} f$ has been made in Theorem 5.1 . 
We now proceed to obtain some estimates for $\|f\|_{(B V)}$. In the first place, from (5.4) we have $|f(x)| \leqq\left[\sup _{0 \leqq t \leqq 1}|x(t)|\right] T_{0}^{1}(g)$ and

$$
\frac{|f(x)|}{\|x\|} \leqq \frac{\sup |x|}{\int_{0}^{1}|x|+T_{0}^{1}(x)} T_{0}^{1}(g) \leqq T_{0}^{1}(g),
$$

from which follows the bound asserted in the theorem. Secondly, for $0 \leqq t_{1}<t_{2} \leqq 1$, we have

$$
\begin{aligned}
\left|g\left(t_{1}\right)-g\left(t_{2}\right)\right| & =\left|f\left(\xi_{t_{1}}-\xi_{t_{2}}\right)\right| \leqq\|f\|_{(B V)} \cdot\left\|\xi_{t_{1}}-\xi_{t_{2}}\right\| \\
& =\|f\|_{(B V)} \cdot\left[\int_{0}^{1}\left(\xi_{t_{2}}-\xi_{t_{1}}\right) d t+T_{0}^{1}\left(\xi_{t_{2}}-\xi_{t_{1}}\right)\right],
\end{aligned}
$$

which implies

$$
\frac{1}{3} \underset{0 \leqq t \leqq 1}{\operatorname{osc}} g(t) \leqq\|f\|_{(B V)} .
$$

Thirdly, we observe that for $x(t) \equiv 1$ on $(0,1)$ we have

$$
f(x)=f\left(\xi_{1}\right)=g(1)=\int_{0}^{1} d g=g(1)-g(0),
$$

which implies $g(0)=0$. Finally, one may determine estimates for $\|f\|_{(B V)}$, which may or may not be closer, by making use of the relation

$$
\int_{0}^{1} x(t) d g=x(1) g(1)-\int_{0}^{1} g(t) d x .
$$

From this we have

$$
\frac{|f(x)|}{\|x\|}=\frac{|f(x)|}{\int_{0}^{1}|x|+T_{0}^{1}(x)} \leqq|g(1)| \frac{|x(1)|}{\int_{0}^{1}|x|+T_{0}^{1}(x)}+\max _{0 \leqq i \leq 1}|g(t)|,
$$

whence follows

$$
\|f\|_{(B V)} \leqq|g(1)|+\max _{0 \leqq t \leqq 1}|g(t)| .
$$

At the same time from the relations

$$
\begin{aligned}
& |g(1)|=\left\|f\left(\xi_{1}\right)\right\| \leqq\|f\|_{(B V)} \cdot\left\|\xi_{1}\right\|=\|f\|_{(B V)}, \\
& |g(t)|=\left\|f\left(\xi_{t}\right)\right\| \leqq\|f\|_{(B V)} \cdot\left\|\xi_{t}\right\|<2\|f\|_{(B V)},
\end{aligned}
$$

we obtain

$$
\max \left[|g(1)|, \quad \frac{1}{2} \max _{0 \leqq t \leqq 1}|g(t)|\right] \leqq\|f\|_{(B V)}
$$


and it may be noted that this implies $\|f\|_{(B V)} \geqq K / 3$, where $K$ stands for the right-hand member of (5.5).

THEOREM 5.3. Every additive and uniformly continuous functional $f(x)$ defined on the space $(C B V)[(A C)]$ can be expressed in the form (5.1) where $\alpha(t)$ is summable, bounded, and independent of $x(t)$, and $\bmod f=B$ as before.

Proof. It has already been observed in $\$ 1$ that $(C B V)$ is dense in $(B V)$. Let $x$ be any point in $(B V)-(C B V)$ and $x_{n}$ any sequence $\varepsilon(C B V)$ such that $\left(x_{n}, x\right) \rightarrow 0$. If the functional $f(x)$ is uniformly continuous on $(C B V), \lim f\left(x_{n}\right)$ exists and we may define $f(x)$ as this limit. Moreover this limit is independent of the sequence $x_{n}$ employed and $f(x)$ thus extended over $(B V)$ is uniformly continuous over $(B V)$. That the extended functional is additive on $(B V)$ may readily be seen as follows. Let $x$ and $y$ be any two points of $(B V)$ at least one of which is not in $(C B V)$. A sequence of sets $S_{n}$ in $(0,1)$ can be so determined that the polygonal functions $x_{n}(t), y_{n}(t)$, and $z_{n}(t)$ respectively inscribed in $x, y$, and $x+y$, whose "corners" are determined by $S_{n}$, converge in our metric to $x, y$, and $x+y$. We then have

$$
\begin{gathered}
z_{n}(t)=x_{n}(t)+y_{n}(t) \text { for all } n, t ; \quad f\left(z_{n}\right)=f\left(x_{n}\right)+f\left(y_{n}\right), \\
\lim f\left(x_{n}\right)=f(x), \quad \lim f\left(y_{n}\right)=f(y), \quad \lim f\left(z_{n}\right)=f(x+y),
\end{gathered}
$$

whence $f(x+y)=f(x)+f(y)$. That $\bmod f=B$ can be shown essentially as in the proof of Theorem 5.1, by appropriately smoothing off the function $\phi(t)$ (as it was designated there) to make it continuous. A similar proof will suffice for the space $(A C)$.

BROWN UNIVERSITY,

Providence, R. I. 